\title{
3D Map Creation Based on Knowledgebase System for Texture Mapping Together with Height Estimation Using Objects' Shadows with High Spatial Resolution Remote Sensing Satellite Imagery Data
}

\author{
Kohei Arai ${ }^{1}$ \\ Graduate School of Science and Engineering \\ Saga University \\ Saga City, Japan
}

\begin{abstract}
Method for 3D map creation based on knowledgebase system for texture mapping together with height estimation using objects' shadows with high spatial resolution of remote sensing satellite imagery data is proposed. Through the experiments with IKONOS imagery data, the proposed method is validated.
\end{abstract}

Keywords-texture mapping; remote sensing satellite; 3D map; object height estimation;

\section{INTRODUCTION}

3D geographic maps are available for public use such as Google map. 3D scenery of major intersections, in particular, is needed to be created for public use. In order to create $3 \mathrm{D}$ scenery of image, cameras mounted on automobiles are used for acquisition of 3D images. It requires huge resources, human resources, time consumable computational resources, and so on. This is referred to the conventional method.

On the other hand, there is the method for 3D map creation with high spatial resolution of remote sensing satellite images, such as Early Bird, Quick Bird ${ }^{1}$ [1], OrbView ${ }^{2}$, IKONOS ${ }^{3}$, etc. Using these satellite imagery data, 3D image can be created. Essentially, satellite acquires images from space with off nadir angle (observation angle). Therefore, top view of 3D object can be acquired while side views are difficult to acquire. Some portion of side view images, however, can be acquired depending on off nadir angle. From the acquired side view images, knowledge about side view image, in particular, texture information can be acquired. Using such knowledge, it is possible to make a texture mapping to the $3 \mathrm{D}$ map. This is referred to the proposed method based on knowledgebase system.

Meanwhile, it is also possible to estimate the objects' height in concern. Using the acquisition time and location of the pixel in concern, then solar zenith angle is calculated. Then objects' height can be estimated with the estimated solar zenith angle and the length of the objects' shadow. The height information estimated by the aforementioned method can be

\footnotetext{
${ }^{1}$ http://www.satimagingcorp.com/gallery-quickbird.html

${ }^{2}$ http://ja.wikipedia.org/wiki/GeoEye

${ }^{3}$ http://ja.wikipedia.org/wiki/GeoEye\#IKONOS
}

used for a confirmation of 3D view of the object in concern. The proposed method uses this procedure of the confirmation of objects' height.

The following section describes the proposed 3D map creation based on knowledgebase system together with the height estimation and confirmation followed by some experiments with IKONOS imagery data. Then conclusion is described together with some discussions.

\section{PROPOSED METHOD}

\section{A. Conventional Method}

3D maps are widely used in public domain. Google map is the most popular 3D map. In order to create 3D maps, cameras mounted vehicles are mostly used. There are major instruments which allow acquisition of 3D images, range cameras, stereoscopic cameras, etc. In Japan, stereoscopic cameras mounted vehicles are used for acquisition of 3D images at around 5400 of major intersections in the relatively large cities every year. It takes one year to update the created 3D image maps in Japan.

In order to save the time required for creation of 3D maps, aerial laser profilers as well as high resolution of remote sensing satellite imagery data are used. The former is to estimate the height of objects, essentially. The later can acquire not only height information but also off nadir view of images are acquired. Therefore, there is a possibility to create 3D maps with such high spatial resolution of remote sensing satellite images.

There is another method for creation of 3D maps. That is conversion method from existing 2D maps to 3D maps. Merits and demerits of these three methods are as follows,

(1) Stereoscopic cameras:

- Relatively high spatial resolution

- $\quad$ It is hard to create automatically

(2) Laser radars:

- It is easy to create automatically

- Relatively low spatial resolution 
(3) Map conversion:

- $\quad$ It is easy to resample 2D maps to 3D maps

- Re-sampled data is usually distorted and has ambiguity

\section{B. Proposed Method}

One of the purposes of the 3D map creation is pedestrian navigation. Therefore, 3D maps have to be comprehensive visually. This implies that comprehensive maps are better than accurate maps (high geometric fidelity). In other words, geometric fidelity is not needed. Therefore, imitated texture derived from the off nadir view of high resolution remote sensing satellite imagery data is used for assumed side view of 3D maps.

\section{Objects' Height Estimation}

Because pixel location of longitude and latitude as well as elevation is known, and also acquisition time is know, then object's height can be estimated with satellite altitude and with length of shadow. Illustrative view of the method for object's height estimation is shown in Figure 1.

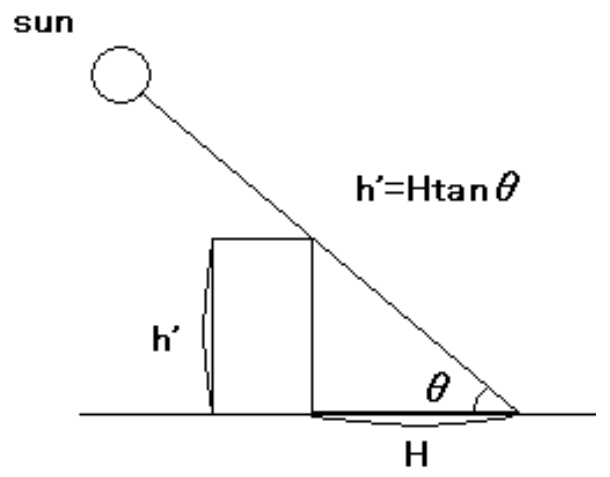

Fig. 1. Object's height estimation

Let $\mathrm{z}$ be solar zenith angle. Then cosine of solar zenith angle can be expressed in equation (1).

$\cos (z)=\sin (a) \sin (b)+\cos (a) \cos (b) \cos (c)$

Where $a, b$, and $c$ denotes latitude of the location in concern, solar latitude, and solar time angle, respectively.

\section{Texture Extraction from the Satellite Images}

Because the acquired high spatial resolution of remote sensing satellite images are distorted and occluded due to the fact that satellite acquires images with off nadir viewing. Therefore, geometric correction with Affine transformation and anti-aliasing is required for extraction of side view of images from the off nadir view of images. Then these images can be used for texture mapping. Occluded portion of side view images can be assumed with knowledge base system.

3D Affine transformation can be expressed as follows,

$$
\left[\begin{array}{l}
x^{\prime} \\
y^{\prime} \\
z^{\prime}
\end{array}\right]=\left[\begin{array}{lll}
v_{1} 1 & v_{1} 2 & v_{1} 3 \\
v_{2} 1 & v_{2} 2 & v_{2} 3 \\
v_{3} 1 & v_{3} 2 & v_{3} 3
\end{array}\right]\left[\begin{array}{l}
x \\
y \\
z
\end{array}\right]+\left[\begin{array}{l}
a \\
b \\
c
\end{array}\right]
$$

Where $(\mathrm{x}, \mathrm{y}, \mathrm{z}) \mathrm{t}$ and $\left(\mathrm{x}^{\prime}, \mathrm{y}^{\prime}, \mathrm{z}^{\prime}\right) \mathrm{t}$ denotes the coordinate before and after the geometric correction. $\mathrm{V}$ matrix denotes magnification, aspect ratio conversion, skew and rotation matrix, and $(\mathrm{a}, \mathrm{b}, \mathrm{c}) \mathrm{t}$ denotes offsets in directions of $\mathrm{x}, \mathrm{y}, \mathrm{z}$.

Due to the fact that affine transformation induces aliasing noises. A single off nadir view of high spatial resolution of satellite image, essentially, would not be enough to create 3D map images. Spatial resolution for side view or opposite side view of images is not good enough for a single off nadir view of the high spatial resolution of remote sensing satellite images. Therefore, anti-aliasing is needed. Anti-aliasing can be done with smoothing filter which allows conversion from zigzag shaped edges to smooth edges.

\section{E. Knowledge Base System}

Occluded portion of side view images have to be assumed with knowledge base system. Also, unclear side view images have to be improved in terms of image quality and have to be assumed some portion of images by using knowledge base system. Knowledge about the artificial constructed objects is as follows,

Knowledge \#1: Because object height can be estimated, the number of floor can be assumed with the knowledge about the floor height.

Knowledge \#2: If windows are aligned on the one side view of the artificial constructed objects, then the opposite side of the artificial constructed objects has same window arrays.

Knowledge \#3: If stairs are attached to one side of the artificial constructed objects, then there is no such stair on the other side of the artificial constructed objects.

\section{EXPERIMENTS}

\section{A. Data Used}

Table 1 shows major specification of IKONOS imagery data which is used for the experiments.

TABLE I. MAJOR SPECIFICATION

\begin{tabular}{|l|l|}
\hline Satellite_Name & IKONOS \\
\hline Provider & Space_Imaging_Co._Ltd. \\
\hline Launch_data & Septembe_25_1999 \\
\hline Mission_life & 7_years \\
\hline Revisit_period & 11_days \\
\hline Inclination & $98.12 \_d e g$. \\
\hline Altitude & $680 \_k m$ \\
\hline
\end{tabular}

Figure 2 shows an example of image of IKONOS. The example image includes shadow of the buildings, unclear side view images of the buildings, one side view image of the buildings, and so on. 


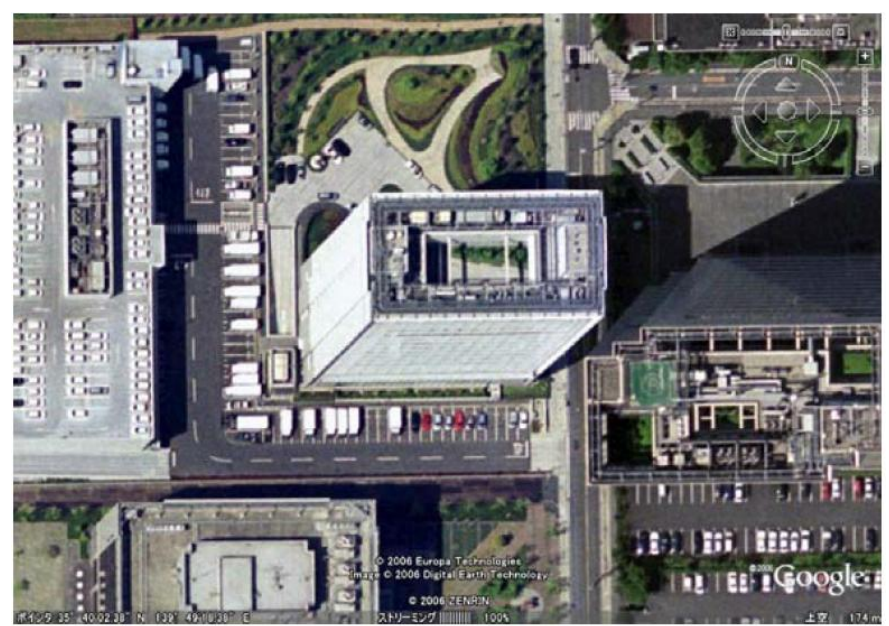

Fig. 2. Example of IKONOS image used for the experiment.

\section{B. 3D Maps from Four Different Aspects of Images Derived from the Single Off Nadir View of KINOS Image}

From the acquired IKONOS image of Figure 2, the following four different aspects of 3D map images are created. Figure 3 (a) shows front view of the created image while Figure 3 (b) shows side view of the created image.

On the other hand, Figure 3 (c) shows rear view of the created image while Figure 3 (d) shows opposite side view of the created image.

The locations of corners of the building are known. Therefore, geometric conversion can be done in accordance with the different viewing angles using Affine transformation. In the created images, there are so much aliasing noises induced by the Affine transformation. It would be better to remove these aliasing noises. Anti-aliasing can be done with smoothing filter which allows conversion from zigzag shaped edges to smooth edges.

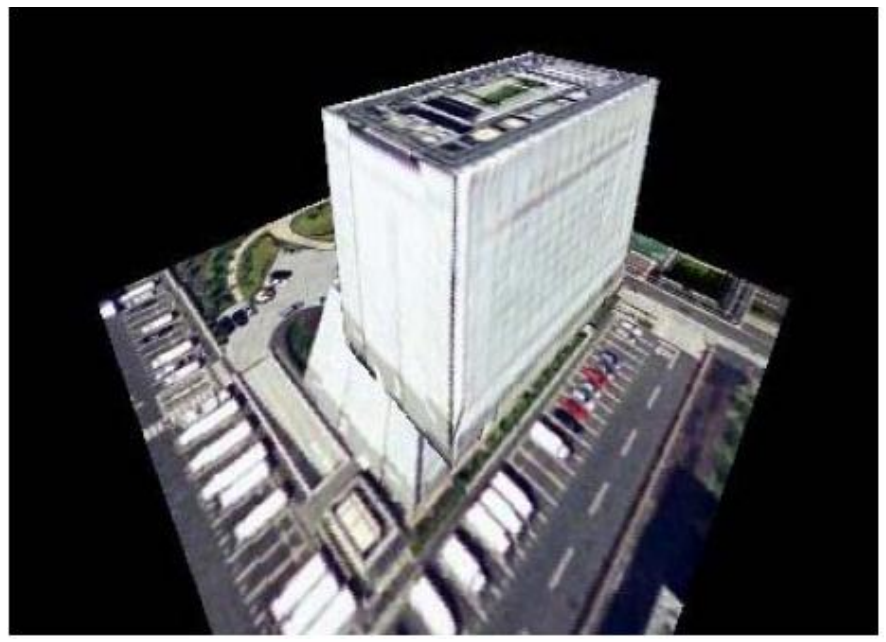

(a)Front view

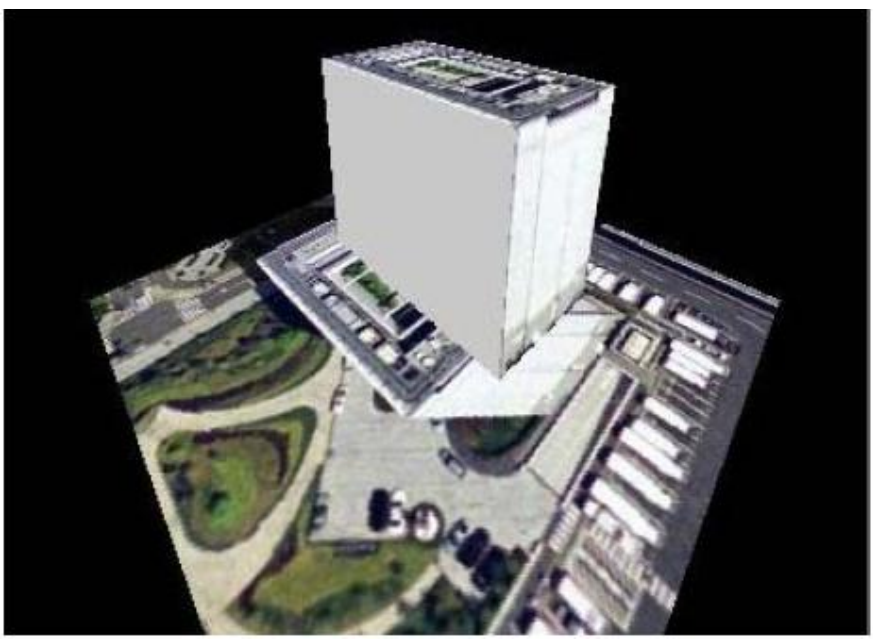

(b)Side view

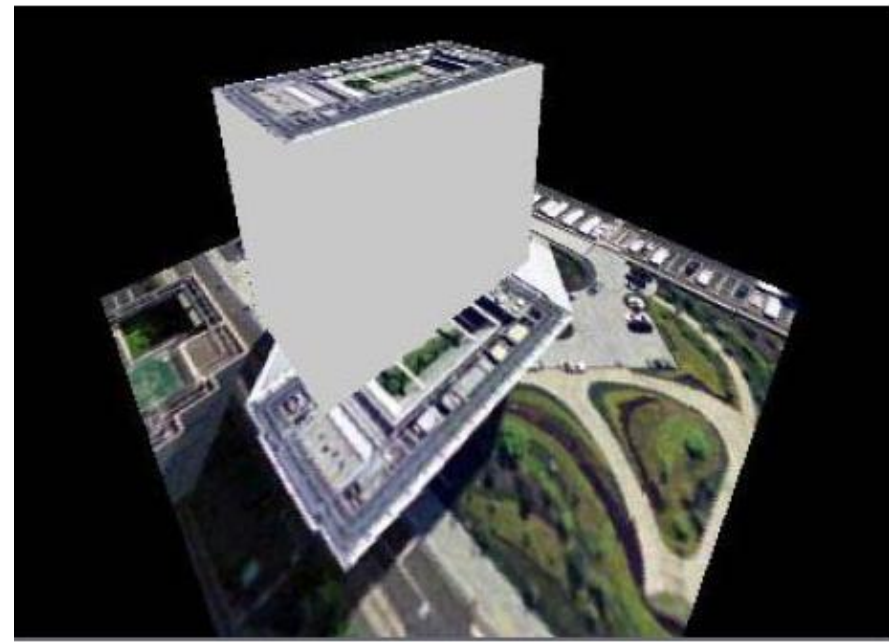

(c)Rear view

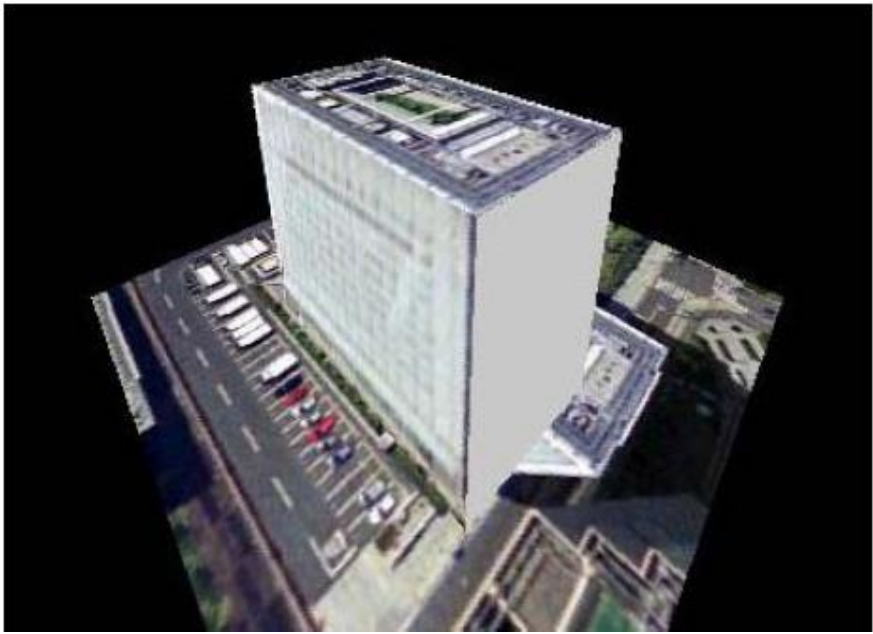

(d)Opposite side view

Fig. 3. Four different aspects of 3D map images derived from a single off nadir view of IKONOS image. 
Moreover, opposite side plane and rear view images cannot be obtained obviously because these planes are occluded. Furthermore, details of the front view and not occluded side view images are unclear due to not enough spatial resolution of IKONOS image for these two planes. Therefore, some image quality improvements are needed for creation of 3D map images. In order to improve the image quality for these planes, the proposed method based on knowledge base system is applied. Firstly, building height is estimated. Then using typical height per one floor $(290 \mathrm{~cm})$ together with the estimated number of floors of the building, texture is derived from the actual acquired IKONOS image. In the same time, anti-aliasing noise reduction is applied to the acquired image to improve image quality.

Front view of 3D map image is used for the rear view of 3D map image. Namely the same images are mapped onto the front and the rear planes of the building. This is one of knowledge (or assumption). This is the same thing for two side view of images. The created texture of the side view of 3D map image derived from the acquired from the IKONOS satellite is mapped onto the opposite side of the building plane which is occluded. Thus 3D map image of four different aspects of images are created. Figure 4 shows the resultant images. In comparison between the created 3D map image of four different aspects of images with (proposed method) and without (the conventional method) consideration of knowledge base system, image quality for the proposed method is superior to that for the conventional method.

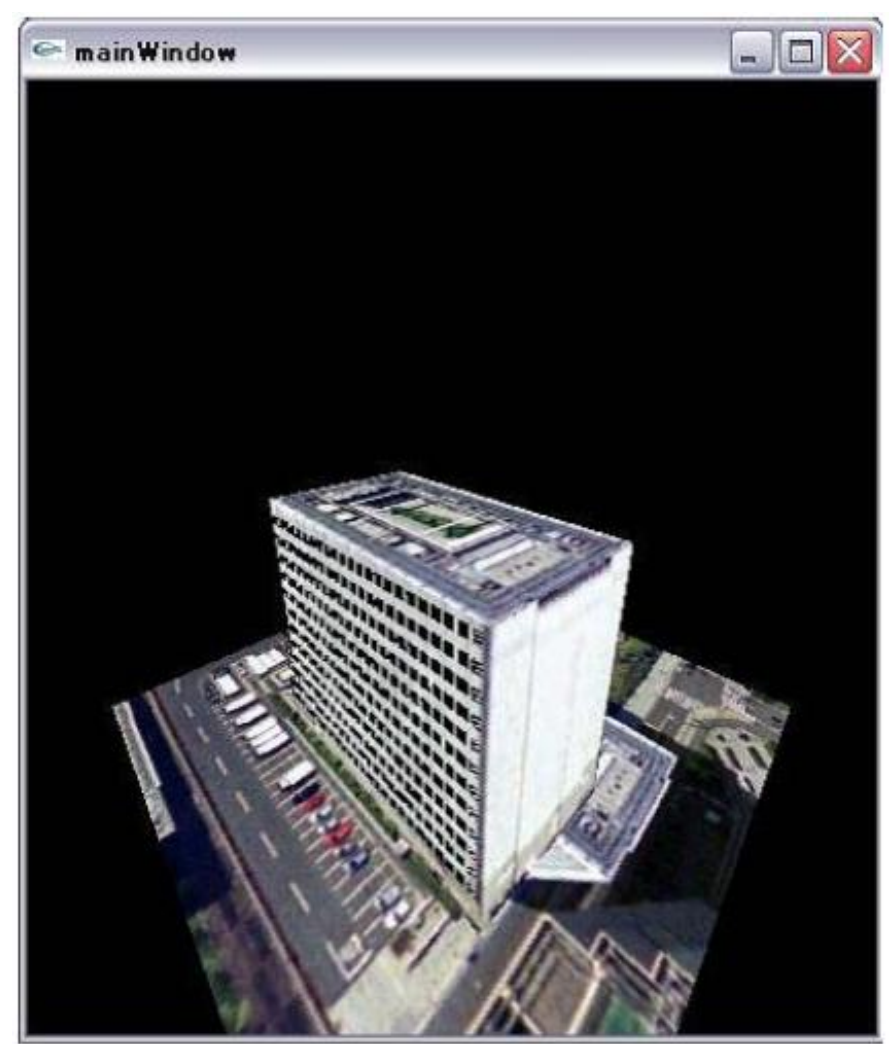

(a)Front view

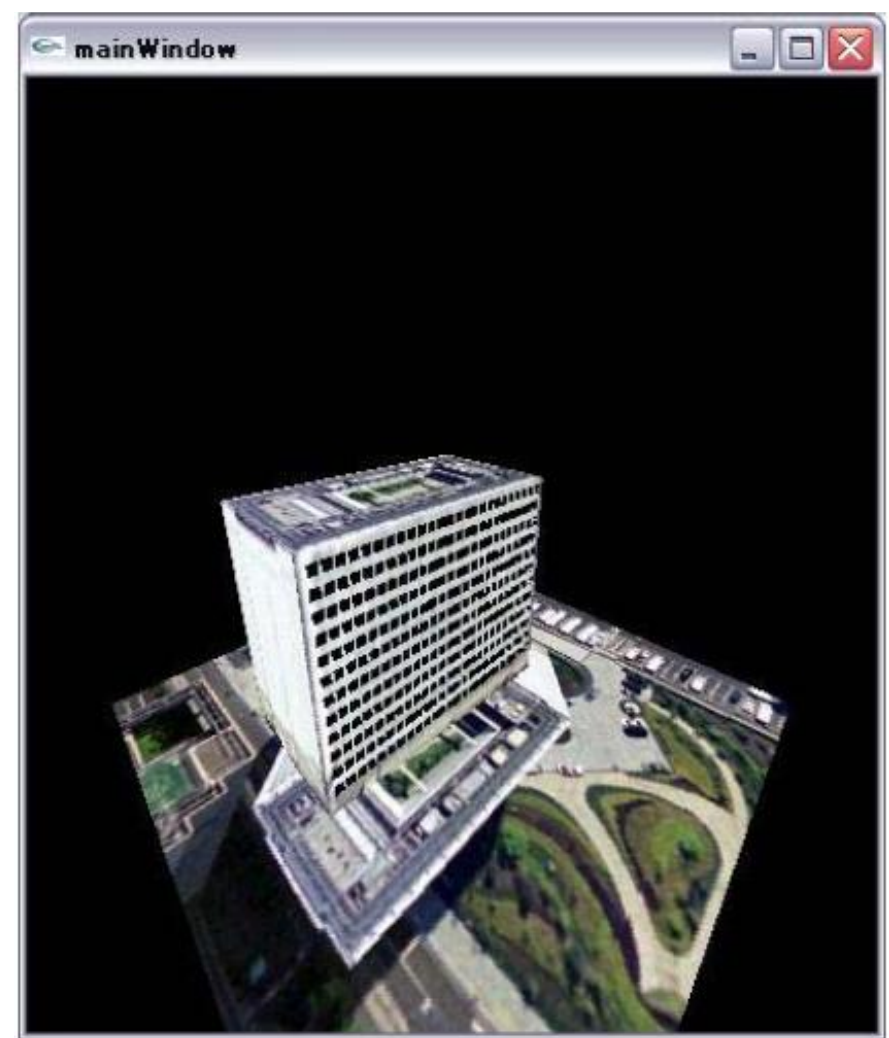

(b)Side view

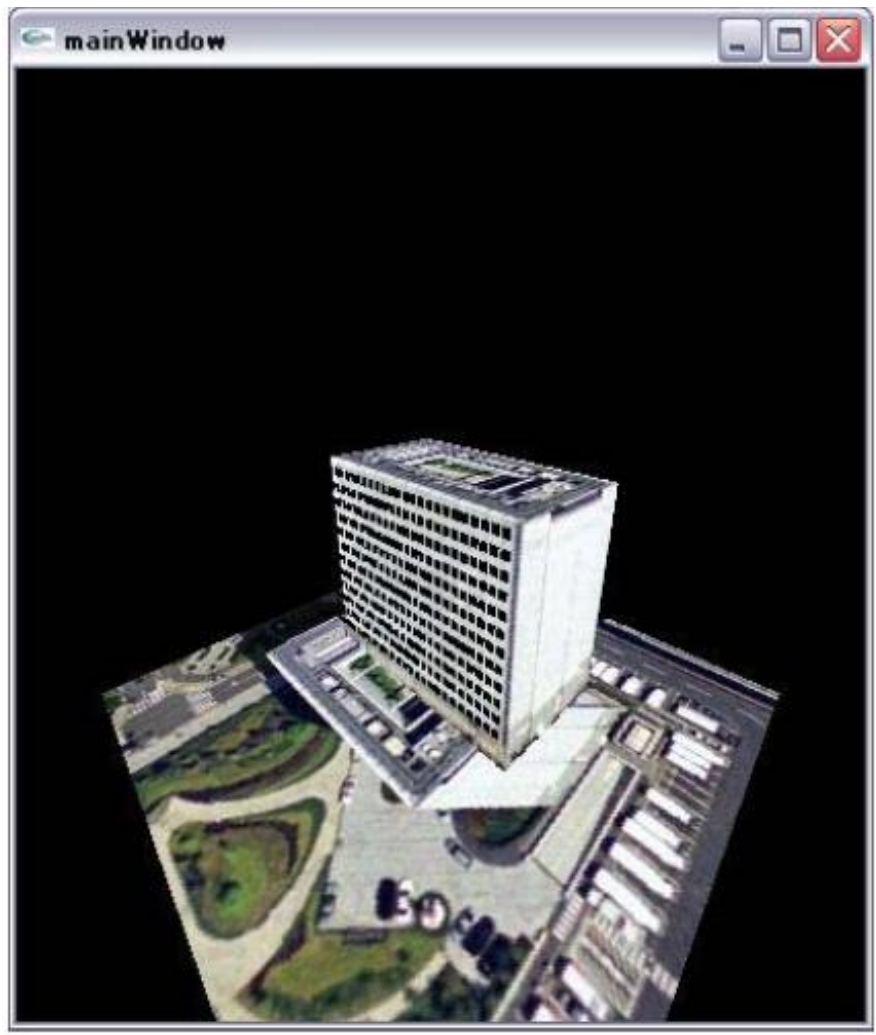

(c)Rear view 


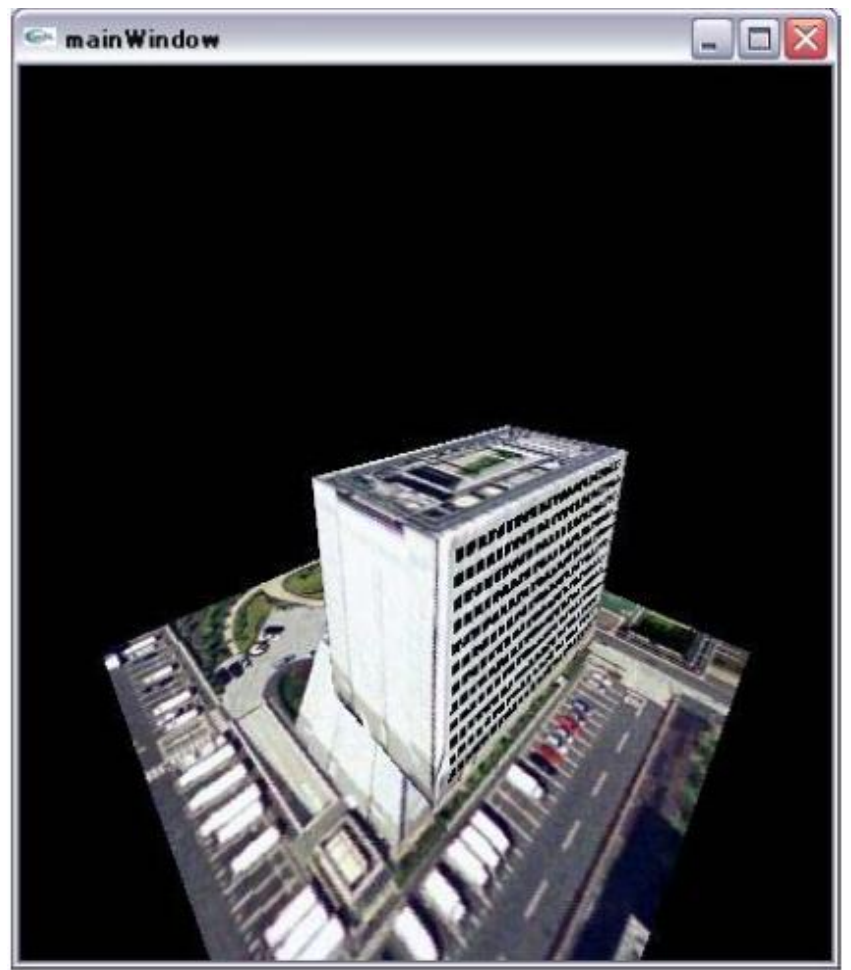

(d)Opposite side view

Fig. 4. Created 3D map image of four different aspects of images

\section{CONCLUSION}

Method for 3D map creation based on knowledgebase system for texture mapping together with height estimation using objects' shadows with high spatial resolution of remote sensing satellite imagery data is proposed. Through the experiments with IKONOS imagery data, the proposed method is validated.

In comparison between the created 3D map image of four different aspects of images with (proposed method) and without (the conventional method) consideration of knowledge base system, it is found that the image quality for the proposed method is superior to that for the conventional method.

\section{ACKNOWLEDGMENT}

The author would like to thank Mr. Kazunori Kinoshita for his effort to conduct experiments.

\section{REFERENCES}

[1] Mehuron, Tamar A., Assoc. Editor (August 2008). "2008 USAF Space Almanac - Major Civilian Satellites in Military Use". Air Force Magazine (Pub: Air Force Association) 91 (8): pp.49-50. ISSN: 0730$6784 .$.

\section{AUTHORS PROFILE}

Kohei Arai, He received BS, MS and PhD degrees in 1972, 1974 and 1982 , respectively. He was with The Institute for Industrial Science and Technology of the University of Tokyo from April 1974 to December 1978 and also was with National Space Development Agency of Japan from January, 1979 to March, 1990. During from 1985 to 1987, he was with Canada Centre for Remote Sensing as a Post Doctoral Fellow of National Science and Engineering Research Council of Canada. He moved to Saga University as a Professor in Department of Information Science on April 1990. He was a councilor for the Aeronautics and Space related to the Technology Committee of the Ministry of Science and Technology during from 1998 to 2000. He was a councilor of Saga University for 2002 and 2003. He also was an executive councilor for the Remote Sensing Society of Japan for 2003 to 2005 . He is an Adjunct Professor of University of Arizona, USA since 1998. He also is Vice Chairman of the Commission A of ICSU/COSPAR since 2008. He wrote 30 books and published 332 journal papers. 